\title{
Competencia digital: docentes en formación y resolución de problemas
}

\author{
Mario Grande-de-Prado \\ Ruth Cañón-Rodríguez \\ Sheila García-Martín \\ Isabel Cantón-Mayo \\ Universidad de León. España. \\ mgrap@unileon.es \\ rcanr@unileon.es \\ sgarcm@unileon.es \\ icanm@unileon.es
}

Recibido: 25/12/2019

Aceptado: 14/10/2020

Publicado: 5/7/2021

\section{Resumen}

Esta investigación tiene como objetivo conocer y analizar la autopercepción que el alumnado que inicia sus estudios en el grado de Maestro de Educación Primaria posee en referencia a su competencia electrónica para resolver problemas técnicos en el entorno digital, por lo que constituye un reto educativo relevante, puesto que se trata de un conocimiento imprescindible en la sociedad actual. El presente examen emplea una metodología descriptivo-interpretativa y ex post facto, utilizando para la recogida de datos un cuestionario dirigido al alumnado del primer curso del grado de Maestro de Educación Primaria de una facultad de Educación durante cuatro años consecutivos. A partir de los resultados obtenidos, se puede afirmar que estos docentes en formación se valoran a sí mismos como competentes. Sin embargo, no debemos olvidar que estos resultados solo muestran la autopercepción de los participantes y que en ellos se encuentran diferencias asociadas al sexo, es decir, los varones del estudio se valoran más competentes y parecen sentirse más seguros ante los problemas que puedan surgir con los dispositivos digitales que las mujeres.

Palabras clave: competencia digital; resolución de problemas; formación del profesorado; TIC; educación superior; tecnología digital

Resum. Competència digital: docents en formació i resolució de problemes

Aquesta investigació té com a objectiu conèixer i analitzar l'autopercepció que posseeix l'alumnat que inicia els estudis en el grau de Mestre d'Educació Primària en referència a la seva competència electrònica per resoldre problemes tècnics en l'entorn digital, per la qual cosa aquesta qüestió esdevé un repte educatiu rellevant, atès que es tracta d'un coneixement imprescindible en la societat actual. El present examen fa servir una metodologia descriptivointerpretativa i ex post facto, utilitzant per a la recollida de dades un qüestionari adreçat a l'alumnat del primer curs del grau de Mestre d'Educació Primària d'una 
facultat d'Educació durant quatre anys consecutius. En base als resultats obtinguts, es pot afirmar que aquests docents en formació es valoren a si mateixos com a competents, però no hem d'oblidar que aquests resultats només ens en mostren l'autopercepció i que es troben diferències associades al sexe, és a dir, els homes de l'estudi es consideren més competents i sembla que se sentin més segurs davant dels problemes que puguin sorgir amb els dispositius digitals que les dones.

Paraules clau: competència digital; resolució de problemes; formació del professorat; TIC; educació superior; tecnologia digital

\section{Abstract. Digital competence: teachers in training and troubleshooting}

The aim of this research is to determine and analyze the self-perception of first-year students in the bachelor's degree in primary teacher education regarding their skills to resolve technical problems in the digital environment. Digital competence is essential is today's society and constitutes a significant educational challenge. A descriptive-interpretative and ex post facto methodology was used. Data were collected by means of a questionnaire administered to first-year students over a period of four consecutive years. The results indicate that teacher trainees consider themselves to be digitally competent, although it should be noted that these results only show the students' self-perception. Some differences related to sex have been found. Specifically, the men in the study rated themselves as being more digitally competent and seem to feel more confident about problems that may arise with digital devices than the women.

Keywords: digital competence; troubleshooting; teacher training; ICT; higher education; digital technology

\section{Sumario}

$\begin{array}{ll}\text { 1. Introducción } & \text { 4. Conclusiones } \\ \text { 2. Metodología } & \text { Referencias bibliográficas }\end{array}$

3. Resultados

\section{Introducción}

En la sociedad actual es indudable que las tecnologías digitales o tecnologías de la información y de la comunicación (TIC) son cruciales, al jugar un papel vital en la formación inicial de los educadores (Cabero y Barroso, 2015; De Pablos, 2015; Lázaro y Gisbert, 2015; Martínez y Cebrián, 2019; RodríguezGarcía, Raso y Ruiz-Palmero, 2019). Las tecnologías se han consolidado como tema clave no solo en el presente, sino también en el futuro de esta sociedad altamente tecnificada (Roblizo y Cózar, 2015; Grande, Cañón y Cantón, 2016; Rodríguez-García, Martínez y Raso, 2017).

En este sentido, se consolida como necesidad imperante el desarrollo de la competencia digital, que puede definirse como la habilidad para usar eficazmente la tecnología con el fin de optimizar nuestra vida cotidiana (Rodríguez-García et al., 2019). Dicha competencia precisa de diferentes habilida- 
des cognitivas, tecnológicas y éticas (Calvani, Cartelli, Fini y Ranieri, 2009), y es considerada condición necesaria para la participación plena del individuo en la sociedad (Ferrari, 2013; INTEF, 2014, 2017; Albertos, Domingo y Albertos, 2016).

Diversas investigaciones, tanto a nivel nacional como internacional, se han centrado en el estudio de la competencia digital de los estudiantes y de los maestros en formación, destacando los estudios de Cabezas, Casillas y Pinto (2014), Lázaro y Gisbert (2015), Cantón, Cañón y Grande (2017), Chan, Churchill y Chiu (2017), Bikos, Stamovlasis y Tzifopoulos (2018), Moreno, Gabarda y Rodríguez (2018), García-Martín y Cantón (2019) o López-Gil y Bernal-Bravo (2019). Los resultados permiten afirmar que los maestros en formación encuentran muchas dificultades para aplicar las tecnologías en el aula, lo que coincide con los resultados obtenidos a partir de diferentes metaanálisis de revisión de literatura científica (Rokenes y Krumsvik, 2014).

Asimismo, el estudio de Cabero y Llorente (2006), con una muestra de 2.000 jóvenes españoles, para el que se elaboró y se validó el instrumento titulado "Cuestionario de Competencias tecnológicas de los alumnos de Secundaria y Bachillerato» (COTASEBA), permite concluir que aunque los estudiantes españoles se consideran competentes para navegar por internet, utilizar los procesadores de texto y el correo electrónico, no creen ser aptos para buscar y gestionar la información ni para utilizar las principales herramientas para comunicarse ni para trabajar en equipo. Estas observaciones se pueden matizar teniendo en cuenta el análisis posterior de Gutiérrez-Castillo y Cabero-Almenara (2016), según el cual tienen la competencia, pero muestran un uso de un nivel medio-bajo.

Al mismo tiempo y en sentido contrario, cuando se trata de competencia digital, es preciso tener en cuenta la denominada segunda brecha digital (Castaño, 2008). Una de las variables que correlacionan, con mayor frecuencia, con una baja competencia digital es el sexo (Burnett, 2011; Fernández, Cebreiro y Fernández, 2011; Roig y Pascual, 2012; Pérez y Vílchez, 2012; Chen, Zhou, Tan y Wong, 2013; Cabezas et al., 2014; Roig, Mengual y Quinto, 2015; Nielsen, Rohman y Lopes, 2018; Ahmad, Rafiq y Ahmad, 2018; Joshi et al., 2020). Estos estudios muestran que las mujeres parecen ser menos competentes, tanto en las TIC en general como en el acceso y el uso de Internet (Castaño, 2008), lo cual incluye a las docentes (Erdogan y Sahin, 2010; Roig et al., 2015). Sin embargo, no todos los autores coinciden en esos resultados (Aguaded, Marín-Gutiérrez y Díaz-Parejo, 2015), al no encontrar diferencias asociadas al sexo. En este sentido, es reseñable el estudio de Bikos et al. (2018) con docentes en formación.

La investigación, por tanto, ha arrojado resultados contradictorios, a lo que además ha de sumarse la complejidad de abordar el estudio y el desarrollo de la competencia digital. Por ello, esta investigación pretende conocer y analizar la percepción de los maestros en formación sobre su competencia en dicho ámbito y estudiar las diferencias asociadas a la variable sexo, para lo cual se toma como referente el modelo explicativo DIGCOMP, referido a la competencia 
digital de los docentes y adoptado dentro del Espacio Europeo de Educación Superior (Ferrari, 2013; INTEF, 2014, 2017).

Este modelo divide la competencia digital en subcompetencias (Carretero, Vuorikari y Punie, 2017; Ferrari, 2013; INTEF, 2014, 2017), organizadas en cinco áreas o dimensiones, que, en su mayoría, están relacionadas e interconectadas entre sí:

1. Información: en la que se incluyen la navegación y el filtrado de esta, así como la evaluación y el almacenamiento y la recuperación de la información.

2. Comunicación: con la interacción a través de medios digitales, la compartición de información y contenidos, la participación ciudadana en línea, la colaboración mediante medios digitales y la gestión de la identidad digital.

3. Creación de contenidos: con el desarrollo de contenidos, la integración y la reestructuración, los derechos de autor y las licencias y la programación.

4. Seguridad: en la que se incluye la protección de dispositivos, la salvaguardia de los datos personales y la identidad digital y la protección del entorno.

5. Resolución de problemas: con la resolución de cuestiones técnicas, la identificación de necesidades y respuestas tecnológicas, la innovación y el uso de tecnologías de manera creativa y la identificación de lagunas en la competencia digital.

Asimismo, dentro de estas cinco dimensiones, se recogen 21 competencias (Ferrari, 2013; INTEF, 2014, 2017), que deben ser adquiridas por los docentes durante su formación. En la presente investigación se aborda la dimensión resolución de problemas y se estudian sus correspondientes áreas y competencias asociadas, las cuales se recogen en la tabla 1. Esta dimensión hace referencia a la capacidad del profesorado para identificar necesidades de uso de recursos digitales, para tomar decisiones apropiadas sobre herramientas tecnológicas, para resolver problemas conceptuales empleando recursos digitales, para usar las tecnologías de forma creativa, para resolver problemas técnicos, así como para actualizar su propia competencia y la de otros (INTEF, 2014, 2017). Una capacitación que se ha constituido como un principio básico profesional y fundamental en las sociedades actuales y del futuro.

Como se puede observar (tabla 1), la competencia digital es un fenómeno complejo, multifacético, que ha generado cuantiosa bibliografía y en la que inciden diferentes factores, siendo uno de los más reconocibles el sexo, aunque no todos los estudios se posicionan igual en sus conclusiones. Esa complejidad que el modelo DIGCOMP abarca con sus diferentes dimensiones es abordada en el presente artículo, centrándose en la resolución de problemas. 
Tabla 1. Resolución de problemas en el modelo DIGCOMP (INTEF, 2014, 2017)

\begin{tabular}{ll}
\hline Área & Competencias asociadas \\
\hline $\begin{array}{l}\text { 5.1. Resolución de } \\
\text { problemas técnicos. }\end{array}$ & $\begin{array}{l}\text { Identificar posibles problemas técnicos y resolverlos (desde la solu- } \\
\text { ción de cuestiones básicas hasta la solución de cuestiones más } \\
\text { complejas). }\end{array}$ \\
$\begin{array}{l}\text { 5.2. Identificación } \\
\text { de necesidades } \\
\text { y respuestas } \\
\text { tecnológicas. }\end{array}$ & $\begin{array}{l}\text { Analizar las propias necesidades en términos tanto de uso de } \\
\text { recursos y herramientas como de desarrollo competencial, asignar } \\
\text { posibles soluciones a las necesidades detectadas, adaptar las herra- } \\
\text { mientas a las necesidades personales y evaluar de forma crítica las } \\
\text { posibles soluciones y las herramientas digitales. }\end{array}$ \\
$\begin{array}{l}\text { 5.3. Innovación y uso de } \\
\text { la tecnología } \\
\text { de forma creativa. }\end{array}$ & $\begin{array}{l}\text { Innovar utilizando la tecnología, participar activamente en produc- } \\
\text { ciones colaborativas multimedia y digitales, expresarse de forma } \\
\text { creativa a través de medios digitales y de tecnologías, generar } \\
\text { conocimiento y resolver problemas conceptuales con el apoyo de } \\
\text { herramientas digitales. }\end{array}$ \\
\hline 5.4. Identificación de & $\begin{array}{l}\text { Comprender las necesidades de mejora y actualización de la propia } \\
\text { lagunas en la } \\
\text { competencia digital. } \\
\text { cia digital, estar al corriente de los nuevos desarrollos. }\end{array}$ \\
\hline
\end{tabular}

Fuente: elaboración propia.

\section{Metodología}

La presente investigación tiene como objetivo analizar y conocer la autopercepción que el alumnado del grado de Maestro de Educación Primaria de una facultad de Educación tiene en referencia a la dimensión resolución de problemas dentro de la competencia digital, así como comprobar cuáles son las diferencias asociadas a la variable sexo.

Se ha optado por una metodología no experimental de tipo descriptivointerpretativo y ex post facto (Sevillano y Vázquez, 2015).

Para obtener la muestra se ha partido de un universo formado por el alumnado del primer curso del grado de Maestro de Educación Primaria de una única facultad española durante cuatro cursos consecutivos, entre los años 2012 y 2016, con un total de 492 estudiantes. La muestra ha sido conformada por 329 alumnos, el 69,9\% de los cuales eran mujeres y el 30,1\%, hombres. El estudio ofrece un nivel de confianza del 99\%, con un porcentaje de error del 4,09\%.

Dada la amplitud de la muestra se ha optado por utilizar un único instrumento, tomando como referencia el cuestionario COTASEBA (Cabero y Llorente, 2006). De él se han seleccionado diez ítems claramente relacionados con la resolución de problemas y que se presentan en forma de escala tipo Likert con 11 posibilidades de respuesta (0-10), donde los valores $0-4$ se consideran negativos y los valores entre 5 y 10 , positivos, siguiendo la estructura indicada por los autores del cuestionario original. Estos ítems han quedado distribuidos en dos subdimensiones a partir de las competencias del modelo DIGCOMP (INTEF, 2017). Dentro de este estudio denominaremos de manera abreviada dichas subdimensiones PRO-A y PRO-B, que corresponden a las áreas Resolución de problemas técnicos e Identificación de necesidades y respuestas tecnológicas. 
Su validez fue determinada por un grupo de expertos integrado por cinco profesores universitarios. Después de contactar personalmente con cada uno de los mencionados docentes, se les remitió un correo electrónico explicándoles brevemente los objetivos perseguidos con la investigación, así como el cuestionario a validar, solicitando su buen criterio para valorar la eficacia de la aplicación del cuestionario. Además, se les entregó una plantilla para que evaluaran cada uno de los ítems del cuestionario en cuanto a univocidad, pertinencia y relevancia. Igualmente, se les dejó abierta la posibilidad de realizar observaciones o modificaciones sobre cualquier aspecto relativo al cuestionario en cada uno de los ítems.

Tabla 2. Modelo de parrilla externa de anotaciones para el juicio de expertos

\begin{tabular}{|c|c|c|c|c|c|c|c|c|c|}
\hline \multicolumn{10}{|l|}{ Ítem } \\
\hline & \multicolumn{2}{|c|}{ Univocidad } & \multicolumn{2}{|c|}{ Pertinencia } & \multicolumn{4}{|c|}{ Importancia } & \multirow{2}{*}{$\begin{array}{l}\text { Observaciones } \\
\text { (sugerencias, correcciones) }\end{array}$} \\
\hline & Sí & No & Sí & No & 1 & 2 & 3 & 4 & \\
\hline & & & & & & & & & \\
\hline
\end{tabular}

Fuente: elaboración propia.

Partiendo de las respuestas obtenidas por los expertos, se procedió a la eliminación o a la conservación de los ítems tomando como criterio de eliminación aquellos que no habían obtenido una puntuación de 3 en el apartado de importancia por al menos 3 de los 5 expertos, y que eran no unívocos o no pertinentes, también para 3 de los 5 expertos.

Una vez eliminados los ítems correspondientes y mejorados los indicados por el grupo de expertos, quedó diseñado el modelo final del cuestionario.

Otras dos áreas no han quedado incluidas en este estudio, bien por estar más claramente relacionadas con otra dimensión del DIGCOMP (Creación) o con otra área de Resolución de problemas, tal como se indica en la tabla 3.

Tabla 3. Áreas no contempladas y sus relaciones

\begin{tabular}{ll}
\hline Área de la dimensión DIGCOMP & Relación \\
\hline $\begin{array}{l}\text { 5.3. Innovación y uso de la tecnología de forma crea- } \\
\text { tiva. }\end{array}$ & $\begin{array}{l}\text { Relacionada con creación de conteni- } \\
\text { dos. }\end{array}$ \\
$\begin{array}{l}\text { 5.4. Identificación de lagunas en la competencia } \\
\text { digital. }\end{array}$ & Relacionada con la dimensión 5.2. \\
\hline
\end{tabular}

Fuente: elaboración propia.

Con respecto a los ítems seleccionados, tras un proceso de evaluación sobre su univocidad, validez y pertinencia con cinco expertos universitarios, se seleccionaron los siguientes campos del COTASEBA, tal y como muestra la tabla 4. 
Tabla 4. Ítems y subdimensiones

\begin{tabular}{|c|c|}
\hline Subdimensión & Ítems del COTASEBA seleccionados \\
\hline $\begin{array}{l}\text { Resolución } \\
\text { de problemas } \\
\text { técnicos } \\
\text { (PRO-A). }\end{array}$ & $\begin{array}{l}\text { 4. Tengo conocimientos básicos sobre el funcionamiento de un ordenador } \\
\text { y sus periféricos. } \\
\text { 5. Sé conectar un ordenador y sus periféricos más usuales: impresoras, } \\
\text { escáneres... } \\
\text { 6. Sé conectar equipos de audio, cámaras de vídeo y fotos digitales a los } \\
\text { ordenadores. } \\
\text { 8. Sé usar de forma apropiada combinaciones de teclas para conseguir } \\
\text { signos alfanuméricos y de puntuación desde el teclado. } \\
\text { 9. Soy capaz de instalar y desinstalar programas informáticos en un orde- } \\
\text { nador. } \\
\text { 10. Puedo cambiar los ficheros de formato (convertir un fichero de un tipo } \\
\text { a otro). } \\
\text { 44. Sé utilizar manuales de ayuda en línea. } \\
\text { 51. Comprendo los problemas de compatibilidad entre hardware y software } \\
\text { informático. }\end{array}$ \\
\hline $\begin{array}{l}\text { Identificación } \\
\text { de necesidades } \\
\text { y respuestas } \\
\text { tecnológicas } \\
\text { (PRO-B). }\end{array}$ & $\begin{array}{l}\text { 21. Uso las calculadoras científicas que incluyen los sistemas operativos } \\
\text { para resolver problemas numéricos. } \\
\text { 47. Me siento competente para reconocer dónde es conveniente elabo- } \\
\text { rar grupos de instrucciones y automatizar procesos de uso frecuente } \\
\text { mediante macros, procedimientos de control, uso de fórmulas... }\end{array}$ \\
\hline
\end{tabular}

Fuente: elaboración propia.

Con respecto a la fiabilidad (alfa de Cronbach), se aplica el coeficiente estandarizado, cuyo cálculo está basado en la medida de las correlaciones existentes entre todos los ítems, y se obtiene el coeficiente total de fiabilidad de la prueba y los coeficientes correspondientes a cada uno de los ítems. El nivel de fiabilidad total es alto, concretamente 0,959 .

Finalmente se facilitó al alumnado el cuestionario mediante un enlace a un formulario en Google Form y, tras obtener las respuestas, se analizaron los datos mediante estadística descriptiva, empleando para ello el paquete estadístico SPSS (Statistical Package Social Science) en su versión 24.0.

Para el análisis estadístico se han empleado estadísticos descriptivos, prueba Kolmogorov-Smirnov (normalidad), U de Mann Whitney (no paramétrica) y ANOVA (para profundizar en los resultados).

\section{Resultados}

Para realizar el análisis de esta dimensión se ha partido de las dos subdimensiones incluidas en la misma: Resolución de problemas técnicos (PRO-A) e Identificación de necesidades y respuestas tecnológicas (PRO-B).

\subsection{Resultados descriptivos: subdimensión PRO-A}

En la mayoría se han obtenido medias superiores a 5 en todos los ítems a excepción del ítem 44, referido a la consulta de manuales de ayuda en línea (4,78), y del ítem 51, referido a la comprensión de los problemas de compatibi- 
Tabla 5. Medidas de tendencia central de ítems de la subdimensión PRO-A

\begin{tabular}{lccccccc}
\hline & Media & Mediana & Moda & Desv. & \multicolumn{4}{c}{ Percentiles } \\
\cline { 6 - 9 } & & & & típ. & $\mathbf{2 5}$ & $\mathbf{5 0}$ & $\mathbf{7 5}$ \\
\hline $\begin{array}{l}\text { 4. Conocimientos básicos sobre } \\
\text { ordenador y periféricos. }\end{array}$ & 6,63 & 7,00 & 7 & 1,791 & 5,00 & 7,00 & 8,00 \\
\hline 5. Conectar periféricos. & 7,13 & 7,00 & 8 & 2,070 & 6,00 & 7,00 & 9,00 \\
\hline 6. Conectar cámaras y audio. & 7,72 & 8,00 & 8 & 1,896 & 7,00 & 8,00 & 9,00 \\
\hline 8. Combinaciones de teclas. & 6,41 & 7,00 & 5 & 2,335 & 5,00 & 7,00 & 8,00 \\
\hline 9. Instalar y desinstalar. & 7,23 & 8,00 & 8 & 2,177 & 6,00 & 8,00 & 9,00 \\
\hline 10. Cambiar formatos. & 6,10 & 6,00 & 6 & 2,390 & 5,00 & 6,00 & 8,00 \\
\hline 44. Manuales de ayuda en línea. & 4,78 & 5,00 & 5 & 2,518 & 3,00 & 5,00 & 7,00 \\
\hline 51. Hardware y software. & 4,16 & 4,00 & 5 & 2,513 & 2,00 & 4,00 & 6,00 \\
\hline
\end{tabular}

Fuente: elaboración propia.

lidad entre hardware y software $(4,16)$. Esto parece indicar que los sujetos encuestados se consideran como mínimo moderadamente competentes para realizar todas las demás habilidades de esta subdimensión.

Los ítems mejor puntuados nos indican que el alumnado participante se considera bastante competente para conectar equipos de audio, cámaras de vídeo $y$ fotos digitales a los ordenadores $(7,72)$, instalar y desinstalar programas informáticos en un ordenador $(7,23)$ y conectar un ordenador y sus periféricos más usuales: impresoras, escáneres... $(7,13)$.

Por el contrario, el alumnado encuestado ha señalado que no se considera suficientemente competente $(4,78)$ para realizar consulta de manuales de ayuda en linea (ítem 44), aunque por un escaso margen, ya que otras medidas de tendencia central (mediana y moda) tienen el valor 5; o para comprender los problemas de compatibilidad entre hardware y software $(4,16)$, tal como se recoge en el ítem 51.

De forma general, se observa un alto índice de valoraciones positivas agrupadas (de 5 a 10) en las que destaca el ítem 6 (un 94\%), referido a conectar equipos de audio, cámaras de vídeo y fotos digitales a los ordenadores, mientras que desde la visión contraria, la comprensión de los problemas de compatibilidad entre hardware y software (ítem 51), es el que presenta la valoración negativa más alta del indicador (un 53,2\%), tal como se puede observar en la tabla 5 . A este ítem le corresponde un $14,0 \%$ en el valor 4, complementado por una moda de 5 . 
Tabla 6. Frecuencias de los ítems de la subdimensión PRO-A

\begin{tabular}{lcc}
\hline Ítem & Negativos (0-4) & Positivos (5-10) \\
\hline $\begin{array}{l}\text { 4. Conocimientos básicos sobre ordenador } \\
\text { y periféricos. }\end{array}$ & $10,9 \%$ & $89,1 \%$ \\
\hline 5. Conectar periféricos. & $9,7 \%$ & $90,3 \%$ \\
\hline 6. Conectar cámaras y audio. & $5,5 \%$ & $94,5 \%$ \\
\hline 8. Combinaciones de teclas. & $17,6 \%$ & $82,4 \%$ \\
\hline 9. Instalar y desinstalar. & $10,6 \%$ & $89,4 \%$ \\
\hline 10. Cambiar formatos. & $22,8 \%$ & $77,2 \%$ \\
\hline 44. Manuales de ayuda en línea. & $39,8 \%$ & $60,2 \%$ \\
\hline 51. Hardware y software. & $53,2 \%$ & $46,8 \%$ \\
\hline
\end{tabular}

Fuente: elaboración propia.

Los ítems 4, 5, 6 y 9 ofrecen un perfil similar, con una tendencia a mostrar valoraciones altas (medidas de tendencia central próximas a 7 , valoraciones positivas cercanas o superiores al $90 \%$ ) para la resolución de problemas sencillos. Dentro de la misma dimensión y para este tipo de problemáticas básicas, los ítems 8 y 10 presentan una tendencia positiva pero más suavizada, con valores centrales más cercanos al 6 y respuestas positivas cercanas al $80 \%$.

\subsection{Resultados descriptivos: subdimensión PRO-B}

En esta subdimensión (PRO-B), en la que se engloban los ítems referidos a la identificación de necesidades y respuestas tecnológicas, se han obtenido medias inferiores a 5 en los dos ítems referidos al uso de las calculadoras cientificas que incluyen los sistemas operativos para resolver problemas numéricos (ítem 21) y a la competencia para reconocer dónde es conveniente elaborar grupos de instrucciones y para automatizar procesos de uso frecuente mediante macros, procedimientos de control, uso de fórmulas... (ítem 47), lo cual nos permite considerar al alumnado como poco competente en estas habilidades, siempre desde su propio punto de vista.

Tabla 7. Medidas de tendencia central de los ítems de la subdimensión PRO-B

\begin{tabular}{lccccccc}
\hline Ítem & Media & Mediana & Moda & Desv. típ. & \multicolumn{3}{c}{ Percentiles } \\
\cline { 6 - 8 } & & & & & $\mathbf{2 5}$ & $\mathbf{5 0}$ & $\mathbf{7 5}$ \\
\hline $\begin{array}{l}\text { 21. Calculadoras } \\
\text { científicas. }\end{array}$ & 4,65 & 5,00 & 5 & 2,628 & 3,00 & 5,00 & 6,00 \\
$\begin{array}{l}\text { 47. Automatizar } \\
\text { procesos. }\end{array}$ & 3,18 & 3,00 & 0 & 2,362 & 1,00 & 3,00 & 5,00 \\
\hline
\end{tabular}

Fuente: elaboración propia. 
A la vista de las medias obtenidas en los dos ítems, comprobamos que los encuestados son escasamente competentes para utilizar calculadoras cientificas $(4,65)$ y para automatizar procesos de uso frecuente mediante macros, procedimientos de control, uso de fórmulas... $(3,18)$.

Tabla 8. Frecuencias de los ítems referidos a aspectos básicos de los ítems de la subdimensión PRO-B

\begin{tabular}{lcc}
\hline Ítem & Negativos (0-4) & Positivos (5-10) \\
\hline 21. Calculadoras científicas. & $42,6 \%$ & $57,4 \%$ \\
\hline 47. Automatizar procesos. & $66,9 \%$ & $33,1 \%$ \\
\hline
\end{tabular}

Fuente: elaboración propia.

Esto se complementa al observar las valoraciones agrupadas (tabla 7), en las que comprobamos que un $57,4 \%$ de los encuestados se considera competente para utilizar las calculadoras científicas, mientras que solamente un $33,1 \%$ se considera competente en la automatización de procesos de uso frecuente mediante macros, procedimientos de control y uso de fórmulas, con lo que la moda se sitúa en el valor 0, con un 19,1\%.

\subsection{Análisis de las diferencias asociadas al sexo}

Para comprobar si existen diferencias vinculadas al sexo, se han analizado las desigualdades existentes teniendo en cuenta la realización de pruebas paramétricas o no paramétricas.

Se realizó la prueba de Kolmogorov-Smirnov para medir la «bondad de ajuste», esto es, si la muestra seguía la distribución normal, y se comprobó que todos los ítems de escala rechazaban la hipótesis de la normalidad de la distribución con la prueba Kolmogorov-Smirnov (tabla 9). Si bien esto nos indica la necesidad de usar pruebas no paramétricas, la falta de normalidad no es un problema severo para el ANOVA, prueba bastante robusta ante esta situación (tabla 9).

Sin embargo, se realizó inicialmente la búsqueda de diferencias con pruebas no paramétricas, como la prueba $U$ de Mann Whitney. Además, a fin de profundizar en esos resultados, se empleó el ANOVA para conseguir una mayor profundidad en el análisis de datos (empleando la prueba de Scheffé).

Tras ello, se han resaltado aquellos ítems en los que la significatividad en el ANOVA y en las pruebas no paramétricas es inferior a 0,005. Todos los ítems que daban resultados llamativos en U de Mann Whitney (no paramétrica) también mostraban resultados significativos en el ANOVA. Como puede observarse en la tabla 10, la mayoría de los ítems correlacionaban con la variable sexo. 
Tabla 9. Análisis de la normalidad de la distribución (prueba Kolmogorov-Smirnov)

\begin{tabular}{lc}
\hline \multicolumn{1}{c}{ Nombre abreviado del ítem } & $\begin{array}{c}\text { Normalidad } \\
\text { (Kolmogorov-Smirnov) }\end{array}$ \\
\hline 4. Conocimientos básicos sobre ordenador y periféricos. & 0,000 \\
\hline 5. Conectar periféricos. & 0,000 \\
\hline 6. Conectar cámaras y audio. & 0,000 \\
\hline 8. Combinaciones de teclas. & 0,000 \\
\hline 9. Instalar y desinstalar. & 0,000 \\
\hline 10. Cambiar formatos. & 0,000 \\
\hline 21. Calculadoras científicas. & 0,000 \\
\hline 44. Manuales de ayuda en línea. & 0,000 \\
\hline 47. Automatizar procesos. & 0,000 \\
\hline 51. Hardware y software. & 0,003 \\
\hline
\end{tabular}

Fuente: elaboración propia.

Tabla 10. Ítems relacionados con la variable sexo

\begin{tabular}{lcc}
\hline Ítem & U de Mann Whitney & ANOVA \\
\hline 9. Instalar y desinstalar. & 0,044 & 0,025 \\
\hline 10. Cambiar formatos. & 0,037 & 0,023 \\
\hline 21. Calculadoras científicas. & 0,001 & 0,010 \\
44. Manuales de ayuda en línea. & 0,001 & 0,001 \\
\hline 47. Automatizar procesos. & 0,002 & 0,001 \\
\hline 51. Hardware y software. & 0,000 & 0,000 \\
\hline
\end{tabular}

Fuente: elaboración propia.

Tomando los ítems significativos y partiendo de la comparación de medias derivada del cálculo del ANOVA para la variable sexo, se han analizado las medidas de tendencia central de dichas variables: media y desviación tipica (tabla 11), comprobando que los varones se puntúan más alto que las mujeres.

Los hombres aventajan a las mujeres en seis de los diez ítems referidos a las dos subdimensiones incluidas en las dimensiones Resolución de problemas técnicos (PRO-A) e Identificación de necesidades y respuestas tecnológicas (PRO-B). Por lo tanto, los varones se sienten más competentes ante problemas con dispositivos. 
Tabla 11. Comparación de medias por sexos

\begin{tabular}{|c|c|c|c|c|c|}
\hline \multirow{4}{*}{ 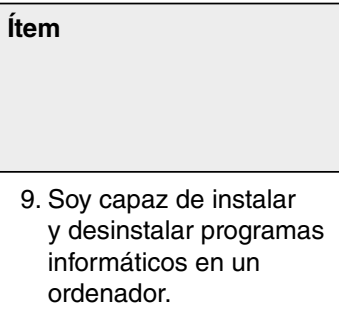 } & \multicolumn{2}{|c|}{ Media } & \multirow{2}{*}{$\begin{array}{c}\text { Desviación } \\
\text { típica } \\
2,244\end{array}$} & \multirow{4}{*}{$\begin{array}{c}\begin{array}{c}\text { Diferencia } \\
\text { entre }\end{array} \\
\begin{array}{c}\text { medias } \\
\text { (hombre/ } \\
\text { mujer) }\end{array} \\
\\
0,59\end{array}$} & \multirow{4}{*}{$\begin{array}{c}\begin{array}{c}\text { Diferencia } \\
\text { entre } \\
\text { desv. típ. } \\
\text { (hombre/ } \\
\text { mujer) }\end{array} \\
\\
0,28\end{array}$} \\
\hline & Mujer & 7,05 & & & \\
\hline & Hombre & 7,64 & 1,961 & & \\
\hline & Total & 7,23 & 2,177 & & \\
\hline \multirow{3}{*}{$\begin{array}{l}\text { 10. Puedo cambiar los } \\
\text { ficheros de formato } \\
\text { (convertir un fichero } \\
\text { de un tipo a otro). }\end{array}$} & Mujer & 5,90 & 2,428 & \multirow{3}{*}{0,66} & \multirow{3}{*}{0,18} \\
\hline & Hombre & 6,56 & 2,246 & & \\
\hline & Total & 6,10 & 2,390 & & \\
\hline \multirow{3}{*}{$\begin{array}{l}\text { 21. Uso las calculadoras } \\
\text { científicas que incluyen } \\
\text { los sistemas operativos } \\
\text { para resolver problemas } \\
\text { numéricos. }\end{array}$} & Mujer & 4,34 & 2,478 & \multirow{3}{*}{1,01} & \multirow{3}{*}{0,36} \\
\hline & Hombre & 5,35 & 2,837 & & \\
\hline & Total & 4,65 & 2,628 & & \\
\hline \multirow{3}{*}{$\begin{array}{l}\text { 44. Sé utilizar manuales de } \\
\text { ayuda en línea. }\end{array}$} & Mujer & 4,48 & 2,511 & \multirow{3}{*}{0,98} & \multirow{3}{*}{0,10} \\
\hline & Hombre & 5,46 & 2,409 & & \\
\hline & Total & 4,78 & 2,518 & & \\
\hline \multirow{3}{*}{$\begin{array}{l}\text { 47. Me siento competente } \\
\text { para reconocer dónde } \\
\text { es conveniente elaborar } \\
\text { grupos de instrucciones } \\
\text { y automatizar proce- } \\
\text { sos de uso frecuente } \\
\text { mediante macros, } \\
\text { procedimientos de con- } \\
\text { trol, uso de fórmulas... }\end{array}$} & Mujer & 2,90 & 2,303 & \multirow[b]{3}{*}{0,93} & \multirow[b]{3}{*}{0,08} \\
\hline & Hombre & 3,83 & 2,382 & & \\
\hline & Total & 3,18 & 2,362 & & \\
\hline \multirow{3}{*}{$\begin{array}{l}\text { 51. Comprendo los proble- } \\
\text { mas de compatibilidad } \\
\text { entre hardware y } \\
\text { software informático. }\end{array}$} & Mujer & 3,73 & 2,415 & \multirow{3}{*}{1,45} & \multirow{3}{*}{0,04} \\
\hline & Hombre & 5,18 & 2,451 & & \\
\hline & Total & 4,16 & 2,513 & & \\
\hline
\end{tabular}

Fuente: elaboración propia.

\section{Conclusiones}

El objetivo de este estudio se ha centrado en conocer la percepción que tienen de sí mismos los maestros en formación sobre cuestiones de resolución de problemas técnicos y tecnológicos. Esto puede plantear algunas cuestiones a tener en cuenta, como hasta qué punto esa autopercepción se ajusta a la realidad o se basa en la confianza de los sujetos en su propia competencia.

A este respecto, hemos podido comprobar que los universitarios que inician sus estudios en el grado de Educación Primaria se sienten muy confiados en sus habilidades para resolver problemas básicos en cuanto a aspectos relacionados con los conocimientos fundamentales de hardware, así como en cuanto a los 
referidos a conectar distintos aparatos, instalar y desinstalar, el uso de atajos o los cambios de formato. Los estudiantes valoran de manera más discreta su propia destreza en el uso de ayudas en línea y en las calculadoras científicas, y se perciben poco hábiles en la automatización de procesos mediante macros $\mathrm{y}$ ante problemas de compatibilidad. Estas observaciones coinciden con otros estudios previos (Cabero y Llorente, 2006; Fernández et al., 2011; Pons y Salinas, 2008).

Respecto a las diferencias en función del sexo, los varones del estudio se consideran más competentes y parecen sentirse más seguros ante los problemas que puedan surgir con los dispositivos digitales que las mujeres. Una vez más, es importante recalcar que estas diferencias no tienen necesariamente que obedecer a una competencia real, aunque pueda derivar en una cierta inseguridad que termine generando una profecía autocumplida. Aun con estas consideraciones, son numerosos los autores que también encuentran diferencias asociadas al sexo (Cabezas et al., 2014; Rokenes y Krumsvik, 2014; Roig et al., 2015) y como consecuencia suelen considerar menos competentes a las mujeres que a los hombres (Castaño, 2008; Erdogan y Sahin, 2010; Roig et al., 2015), aunque no todos los estudios coinciden en esa valoración (Aguaded et al., 2015; Bikos et al., 2018).

Por último, queremos hacer mención a las limitaciones de esta investigación, señalando que somos conscientes de que su aplicación en una única facultad y el uso de un solo instrumento puede ser un hándicap en la generalización de los resultados obtenidos, a pesar de la amplia muestra que ha conformado el estudio y que aporta robustez a los resultados obtenidos.

Por ello, el desarrollo de estudios similares en otros ámbitos geográficos y el uso de diferentes análisis e instrumentos (cualitativos, test de ejecución de tareas) sobre la competencia digital de los futuros maestros pueden suponer un punto de partida importante para afrontar los retos de su formación inicial, debiendo impulsar cambios significativos en la formación de los docentes como pilar básico de la educación actual.

\section{Referencias bibliográficas}

Aguaded, I.; Marín-Gutiérrez, I. y Díaz-Parejo, E. (2015). La alfabetización mediática entre estudiantes de primaria y secundaria en Andalucía (España). RIED: Revista Iberoamericana de Educación a Distancia, 18(2), 275-298. <http://doi.org/10.5944/ried.18.2.13407>

Ahmad, S.; RafiQ, M. y Ahmad, S. (2018). Gender disparities in the use of internet among graduate students of a developing society: A case of Pakistani universities. Global Knowledge, Memory and Communication, 67(4/5), 226-243. $<$ https://doi.org/10.1108/GKMC-11-2017-0092>

Albertos, A.; Domingo, A.. y Albertos, J. (2016). Estrategia docente para el desarrollo de la competencia digital en el aula universitaria: Del uso recreativo al uso formativo. Educar, 52(2), 243-261.

$<$ https://doi.org/10.5565/rev/educar.732> 
Bikos, K.; Stamovlasis, D. y Tzifopoulos, M. (2018). Dimensions of digital divide and relationships with social factors: A study of Greek pre-service teachers. Themes in eLearning, 11(1), 23-34. Recuperado de <http://earthlab.uoi.gr/tel/index.php/ themeselearn/article/view/12/2>.

Burnett, C. (2011). Preservice teachers' digital literacy practices: Exploring contingency in identity and digital literacy in and out of educational contexts. Language and Education, 25(5), 433-449. <http://dx.doi.org/10.1080/09500782.2011.584347>

Cabero, J. y Barroso, J. (coords.) (2015). Nuevos retos en tecnología educativa. Madrid: Síntesis.

Cabero, J. y Llorente, M.C. (2006). La rosa de los vientos: Dominios tecnológicos de las TIC por los estudiantes. Sevilla: GID.

Cabezas, M.; Casillas, S. y Pinto, A.M. (2014). Percepción de los alumnos de Educación Primaria de la Universidad de Salamanca sobre su competencia digital. EDUTEC: Revista Electrónica de Tecnología Educativa, 48. <https://doi.org/10.21556/edutec.2014.48.156>.

Calvani, A.; Cartelli, A.; Fini, A. y Ranieri, M. (2009). Models and instruments for assessing digital competence at school. Journal of ELearning and Knowledge Society-English Version, 4(3).

Cantón, I.; CaÑón, R. y Grande, M. (2017). La comunicación como subdimensión de la competencia digital en futuros maestros de Educación Primaria. Pixel-Bit: Revista de Medios y Educación, 48, 33-47. <https://doi.org/10.12795/pixelbit.2017.i50.02>

Carretero, S.; Vuorikari, R. y Punie, Y. (2017). DigComp 2.1: The Digital Competence Framework for Citizens: With eight proficiency levels and examples of use. Luxemburgo: Publicaciones de la Unión Europea. <https://doi.org/10.2760/38842>

Castaño, C. (2008). La segunda brecha digital. Madrid: Cátedra.

Chan, B.S.; Churchill, D. y Chiu, T.K. (2017). Digital Literacy Learning in Higher Education Through Digital Storytelling Approach. Journal of International Education Research (JIER), 13(1), 1-16. <https://doi.org/10.19030/jier.v13i1.9907>

Chen, W.; Zhou, X.; TAN, A. y Wong, P.S.K. (2013). ICT experiences and competencies of pre-service teachers in the digital age. Recuperado de <https://goo.gl/BTUi$\mathrm{Bf}>$.

Comisión Europea (2006). Competencias clave para el aprendizaje permanente. Recuperado de <http://eur-lex.europa.eu/legal-content/ES/TXT/?uri=URISERV\%3Ac $11090>$.

Díez Gutiérrez, E.J. (2014). Video games and gender-based violence. Procedia-Social and Behavioral Sciences, 132, 58-64.

- (dir.) (2004). La diferencia sexual en el análisis de los videojuegos. Madrid: CIDEInstituto de la Mujer.

Erdogan, A. y Sahin, I. (2010). Relationship between Math Teacher Candidates' Technological Pedagogical and Content Knowledge (TPACK) and Achievement Levels. Procedia-Social Behavioral Sciences, 2, 2707-2711. $<$ https://doi.org/10.1016/j.sbspro.2010.03.400>

Fernández, C.; Cebreiro, B. y Fernández, C. (2011). Competencias para el aprendizaje en red de los alumnos de educación secundaria en Galicia. Pixel-Bit: Revista de Medios y Educación, 38, 7-21. 
Ferrari, A. (2013). DIGCOMP: A Framework for Developing and Understanding Digital Competence in Europe. Luxemburgo: Publicaciones de la Unión Europea. $<$ https://doi.org/10.2788/52966>

García-Del-Castillo, J.A. (2012). Concepto de percepción de riesgo y su repercusión en las adicciones. Health and Addictions, 12(2), 133-151. <https://doi.org/10.21134/haaj.v12i2.2>

García-Martín, S. y Cantón-Mayo, I. (2019). Uso de tecnologías y rendimiento académico en estudiantes adolescentes / Use of technologies and academic performance in adolescent students. Comunicar, 59, 73-81. $<$ https://doi.org/10.3916/C59-2019-07>

García-Martín, J. y García-Sánchez, J.N. (2013). Patterns of Web 2.0 tool use among young Spanish. Computers \& Education, 67, 105-120. $<$ https://doi.org/10.1016/j.compedu.2013.03.003>

Grande-de-Prado, M.; Cañón, R. y Cantón, I. (2016). Competencia digital y tratamiento de la información en futuros maestros de Primaria / Digital competence and information management in future primary school teachers. Educatio Siglo XXI, 34(3), 101-118. $<$ http://dx.doi.org/10.6018/j/275961>

Gutiérrez-Castillo, J.J. y Cabero-Almenara, J. (2016). Estudio de caso sobre la autopercepción de la competencia digital del estudiante universitario de las titulaciones de grado de educación infantil y primaria. Profesorado: Revista de Currículum y Formación del Profesorado, 20(2), 180-199. Recuperado de <http://hdl. handle.net/11441/45324>.

Gutiérrez-Castillo, J.J.; Cabero-Almenara, J. y Estrada-Vidal, L.I. (2017). Diseño y validación de un instrumento de evaluación de la competencia digital del estudiante universitario. Revista Espacios, 38(10), 1-27.

INTEF (2014). Marco Común de Competencia Digital Docente V 2.0. Madrid: Ministerio de Educación, Cultura y Deporte. Recuperado de <http://goo.gl/ DmZA1t>.

- (2017). Marco Común de Competencia Digital Docente V 2.0. Madrid: Ministerio de Educación, Cultura y Deporte. Recuperado de <http://educalab.es/docu ments/10180/12809/MarcoComunCompeDigiDoceV2.pdf >.

Joshi, A.; Malhotra, B.; Amadi, C.; Loomba, M.; Misra, A.; Sharma, S.; Arora, A. y Amatya, J. (2020). Gender and the Digital Divide Across Urban Slums of New Delhi, India: Cross-Sectional Study. Journal of Medical Internet Research, 22(6), e14714.

Lázaro Cantabrana, J. y Gisbert Cervera, M. (2015). El desarrollo de la competencia digital docente a partir de una experiencia piloto de formación en alternancia en el grado de Educación. Educar, 51(2), 321-348. $<$ https://doi.org/10.5565/rev/educar.725>

López-Gil, M. y Bernal-Bravo, C. (2019). El perfil del profesorado en la Sociedad Red: Reflexiones sobre las competencias digitales de los y las estudiantes en Educación de la Universidad de Cádiz. International Journal of Educational Research and Innovation (IJERI), 11, 83-100.

Martínez Romera, D. y Cebrián Robles, D. (2019). Análisis videográfico para la evaluación de los aprendizajes en las prácticas externas de la formación inicial de docentes de secundaria. Educar, 55(2), 457-477. $<$ https://doi.org/10.5565/rev/educar.873> 
Nielsen, M.; Rohman, I. y Lopes, N. (2018). Empirical analysis of the current digital divides since 2010. Proceedings of the 11th International Conference on Theory and Practice of Electronic Governance (pp. 616-625). Irlanda: ACM.

Moreno, M.; Gabarda, V. y Rodríguez, A. (2018). Alfabetización informacional y competencia digital en estudiantes de Magisterio. Profesorado: Revista de Currículum y Formación del Profesorado, 22(3), 253-270. <https://doi.org/10.30827/profesorado.v22i3.8001>

Pablos Pons, J. de (coord.) (2015). Los centros educativos ante el desafío de las tecnologías digitales. Madrid: La Muralla.

Pérez, F. y Vílchez, J.E. (2012). El uso de los videojuegos y redes sociales como predictores de la integración curricular de las TIC en estudiantes de Magisterio. Sphera Pública, 12, 199-215. Recuperado de <https://www.redalyc.org/articulo. oa?id=29729577013>.

Pons, B. y Salinas, J. (2008). Competències tecnològiques dels alumnes i professors de les Illes Balears: Anuari de l'educació de les Illes Balears. Mallorca: Universitat de les Illes Balears.

Prensky, M. (2001). Digital Natives, Digital Inmigrants. On the Horizon, 9(5), 1-6. <https://doi.org/10.1108/10748120110424816>

- (2011). Enseñar a nativos digitales. Madrid: SM.

Roblizo, M.J. y Cózar, R. (2015). Usos y competencias en TIC en los futuros maestros de educación infantil y primaria: Hacia una alfabetización tecnológica real para docentes. Pixel-Bit: Revista de Medios y Educación, 47, 23-39. <https://doi.org/10.12795/pixelbit.2015.i47.02>

Rodríguez-García, A.M.; Martínez, N. y Raso, F. (2017). La formación del profesorado en competencia digital: Clave para la educación del siglo xxi. Revista Internacional de Didáctica y Organización Educativa, 3(2), 46-65.

Rodríguez-García, A.M.; Raso Sánchez, F. y Ruiz-Palmero, J. (2019). Competencia digital, educación superior y formación del profesorado: Un estudio de metaanálisis en la Web of Science. Pixel-Bit: Revista de Medios y Educación, 54, 65-82. <http://dx.doi.org/10.12795/pixelbit.2019.i54.04>

Roig, R. y Pascual, A.M. (2012). Las competencias digitales de los futuros docentes: Un análisis con estudiantes de Magisterio de Educación Infantil de la Universidad de Alicante. @tic: Revista d'Innovació Educativa, 9, 53-60. <https://doi.org/10.7203/attic.9.1958>

Roig Villa, R.; Mengual Andrés, S. y Quinto Medrano, P. (2015). Conocimientos tecnológicos, pedagógicos y disciplinares del profesorado de Primaria. Comunicar: Revista Científica de Comunicación y Educación, 23(45), 151-159. <http://dx.doi.org/10.3916/C45-2015-16>

Rokenes, F. y Krumsvik, R.J. (2014). Development of Student Teachers' Digital Competence in Teacher Education - A Literature Review. Nordic Journal of Digital Literacy, 4, 250-280. $<$ https://doi.org/10.18261/ISSN1891-943X-2014-04-03>

Sevillano, M.L. y Vázquez Cano, E. (2015). Modelos de investigación en contextos ubicuos y móviles en educación superior. Madrid: McGraw-Hill y UNED. 